\title{
Vintage Physiology
}

\section{Otto Warburgs „,Labor-Kochbücher" und Apparaturen}

\author{
Mathias Grote
}

\begin{abstract}
Vintage Physiology. Otto Warburg's Laboratory Manuals and Instruments
Laboratory manuals, notebooks and instrumentation from Otto Warburg's Institute of Cell Physiology have been discovered at the Max Planck Institute of Biochemistry. Following Warburg's death in 1970 one of his technicians was transferred from Berlin-Dahlem to the newly founded biochemical institute at Martinsried near Munich, and brought with him these resources, which epitomize the singular position of Warburg's work style and institute in the 1950s and 1960s. Since the technician's skills and apparently outmoded instrumentation like the Warburg apparatus were still used in the context of 1970s membrane biochemistry, this trove also documents an unexpected continuity of research within the life sciences. Moreover, the service of Warburg's technician, which spans more than four decades, as well as the meticulous documentation of preparations and experiments, serve as a case in point for the importance of routine and rather mundane techniques in the post-war molecular life sciences.
\end{abstract}

Keywords: Otto Warburg, Max Planck Institute, biochemistry, cell physiology, technician, laboratory manuals

Schlüsse/wörter: Otto Warburg, Max-Planck-Institut, Biochemie, Zellphysiologie, technischer Assistent, Laborbücher

\section{Von Vorgestern: Warburg in Martinsried, 2012}

Die Max-Planck-Institute (MPI) für Biochemie und Zellphysiologie stellen sinnfällig den Kontrast zwischen grundverschiedenen Stadien der Lebenswissenschaften im zwanzigsten Jahrhundert dar. Hier ein flacher Betonbau, 1972 in Martinsried bei München auf freiem Feld eingeweiht, dort Otto Warburgs Dahlemer Institut, erbaut Anfang der 1930er Jahre im Stil eines märkischen Gutshauses und heute Sitz des Archivs der Max-Planck-Gesellschaft (MPG; siehe Abb. 1).

Auch mit Hinblick auf den Forschungsbetrieb scheinen die Differenzen offensichtlich: In Dahlem ein „klassisches Ein-Mann-Institut “1 , in dem Warburg und seine Mitarbeiter seit den 1930er Jahren etwa Photosynthese- und Krebsforschung betrieben, dort eines der größten Institute der MPG, mit 

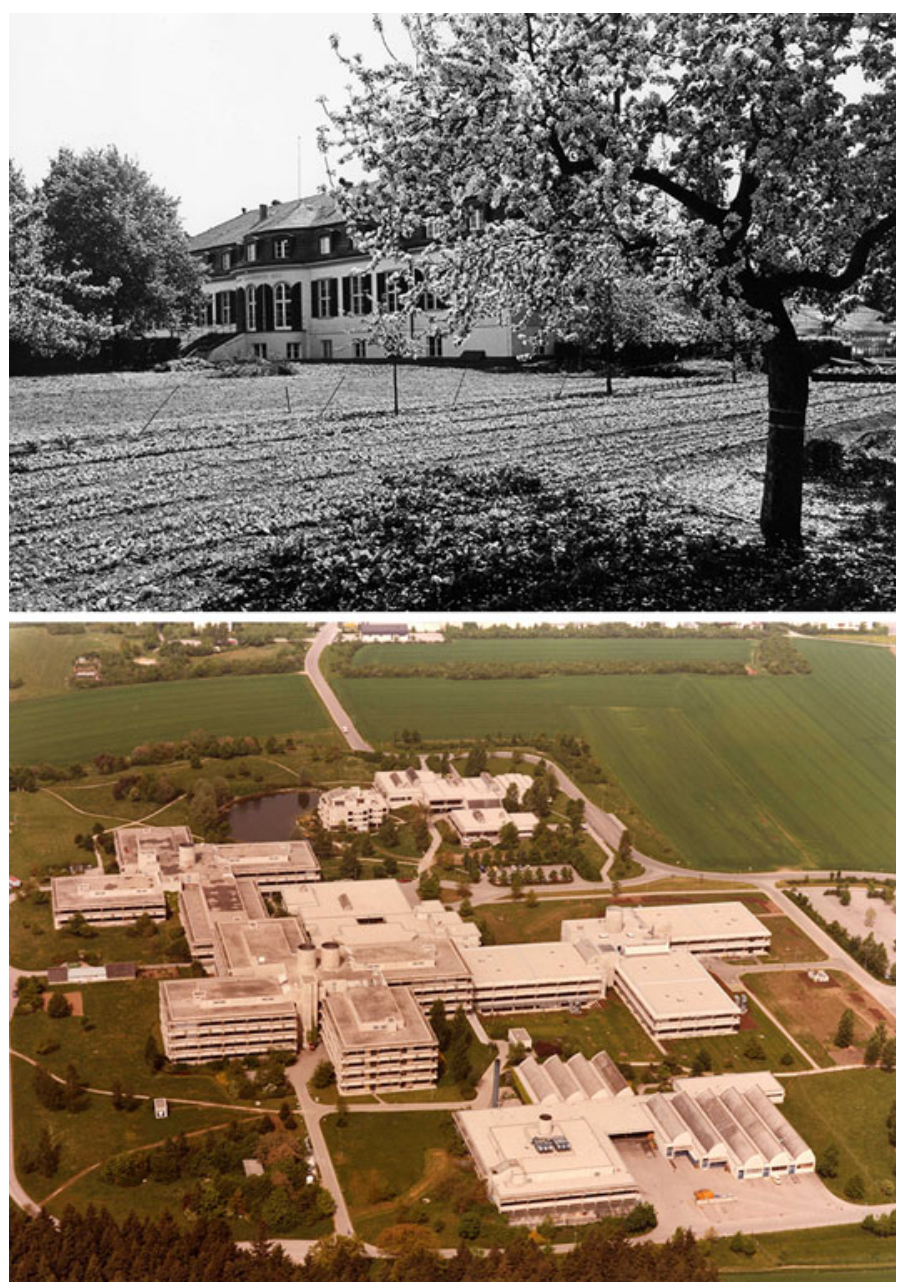

Abb. 1 Die Max-Planck-Institute für Biochemie in Martinsried und Zellphysiologie in BerlinDahlem. ${ }^{2}$ Luftaufnahme: Copyright Bavaria Luftbild GmbH, Freigabe-Nr. 2844. Beide Aufnahmen veröffentlicht mit freundlicher Genehmigung des Archivs der MPG, BerlinDahlem

anfangs elf Abteilungen, die etwa zu Elektronenmikroskopie, Proteinstrukturoder Virusforschung arbeiteten.

Überraschend oder nicht, in der von dem Biochemiker Dieter Oesterhelt geleiteten Martinsrieder Abteilung "Membranbiochemie" fanden sich im Rahmen einer historischen Erforschung der Lebenswissenschaften nach 1970 Spuren einer Kontinuität zwischen diesen unterschiedlichen Epochen der Wissenschaft. Im Keller der Abteilung lagerten aus Holz gefertigte Halterungen und Glasgefäße, die Bestandteile so genannter Warburg-Apparaturen darstellten, einem seit den 1920er Jahren klassischen Instrument zur Bestimmung der Gasentwicklung von Geweben oder Zellsuspensionen. Die 
Schränke enthielten ferner optische Bänke und Filter sowie ein in Berlin gefertigtes Bolometer, welches vor der Einführung von Halbleitertechnologien zur Quantifizierung von Strahlungsintensitäten genutzt wurde (Abb. 2).

Das Verbindungsglied zwischen Martinsried und Dahlem bildete der Labortechniker Günter Krippahl, der in beiden Instituten angestellt war und dessen im Münchner Umland lebender Sohn auf Nachfrage einen Karton mit Laborbüchern anlieferte, welche fast zwanzig Jahre von Krippahls Arbeit im Institut für Zellphysiologie dokumentieren. Außerdem besaß Oesterhelt bereits drei schwere Handbücher - Warburgs „Kochbücher“, die er von Krippahl kurz vor dessen Tod in den 1990er Jahren erhalten hatte. ${ }^{3}$

Auf welchem Wege gelangten Krippahl sowie diese Instrumente und Unterlagen nach Martinsried, und vor allem, welche Rolle spielten diese zellphysiologischen Verfahren in einem Institut, das just in dem Jahr den Betrieb aufnahm als das Dahlemer aufgelöst wurde? Wie ist die Verbindung der Zellphysiologie der Vorkriegszeit zur Membranbiochemie der 1970er

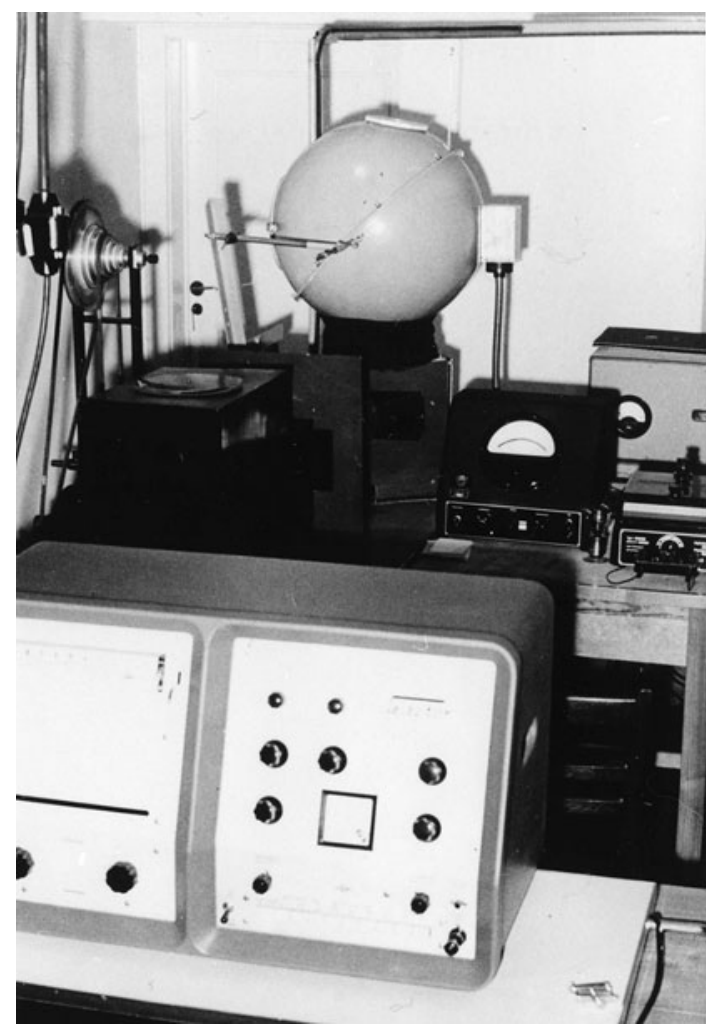

Abb. 2 Experimenteller Aufbau zur Messung von Strahlungsintensitäten mit einer Ulbricht'schen Kugel und einem Bolometer in Warburgs Institut, 1962. Derartige Instrumente wurden am MPI für Biochemie zur Untersuchung der das Bakteriorhodopsin enthaltenden Halobakterien verwandt. ${ }^{4}$ Veröffentlicht mit freundlicher Genehmigung des Archivs der MPG, Berlin-Dahlem 
Jahre - dem ursprünglichen Forschungsinteresse des Autors - zu verstehen, die auch in einer gemeinsamen Publikation von Oesterhelt und Krippahl dokumentiert ist? Der Anachronismus von Warburg in Martinsried, oder anders ausgedrückt, die Koexistenz verschiedener Zeitschichten der Wissenschaft, die sich in diesem Fund materialisiert, scheint ein ungewohntes Licht auf die Geschichte der molekularen Lebenswissenschaften zu werfen, insbesondere da deren Wahrnehmung durch sich in der Nachkriegszeit rasch entwickelnde Felder wie Genetik und Strukturforschung dominiert ist (Morange 1998, Olby 1990).

\section{Kontinuität und Kuriosum: Zellphysiologie in Berlin-Dahlem, 1950-1972}

Ein Blick in Warburgs Bücher macht schnell klar, dass diese nicht nur „Koch-“ oder genauer Protokollbücher darstellten - es finden sich etwa Präparationsvorschriften chemischer Substanzen, Anleitungen zur Durchführung bestimmter Experimente oder Beschreibungen von Apparaturen - sondern ein vielschichtiges Referenzsystem, in dem Warburg auch Personen, Literaturverweise oder summarische Darstellungen von Theorien handschriftlich vermerkte. Während etwa der Eintrag „Aepfelsäure" spezielle Eigenschaften dieses Zwischenproduktes des Intermediärstoffwechsels zusammenfasst, beschreibt „Ascites-Tumoren“, wie Proben dieses Modellsystems der Krebsforschung präpariert wurden. ${ }^{5}$ Unter „Brunnenwasser“ stößt der Leser auf die mineralische Zusammensetzung des Wassers aus dem Dahlemer Institutsbrunnen, der Eintrag „Chlorella“ beschreibt die Anzucht dieser einzelligen Grünalge für Photosyntheseversuche und „Fett für Manometer“ spezifiziert, mit welchem Schmierstoff die Schliffstopfen von Glasgefäßen der WarburgApparaturen gasdicht gemacht wurden. Die Bezeichnung „Kochbuch“ erklärt sich beispielsweise aus der Präparationsvorschrift für das so genannte „Diphospho-pyridin-nukleotid“ (DPN), eines von Warburg in den 1930er Jahren charakterisierten Coenzyms: Man zerbröckele $20 \mathrm{~kg}$ Bäckerhefe, mische sie mit 201 Phenol, rühre, zentrifugiere und filtriere, versetze die Lösung mit 321 Äther, um nach vielen weiteren Schritten einige Gramm eines Trockenpräparates zu erhalten - kurzum, der Eintrag zeigt die vermutlich in Warburgs Institut etablierten, handwerklichen Routinen, die nötig waren, um Material für bestimmte biochemische Untersuchungen zu gewinnen.

Da weder Papier noch Einband der Bücher Spuren von Laborarbeit aufweisen, kann vermutet werden, dass die drei Bände eine Schnittstelle zwischen dem Institut und anderen Orten der wissenschaftlichen Arbeit darstellten etwa einem Schreibzimmer in Warburgs Ferienhäusern. Obwohl die meisten Eintragungen von Warburg selbst sorgfältig vorgenommen wurden, finden 
sich auch einige Protokolle in den Handschriften seiner Mitarbeiter sowie gelegentlich eingeklebte Produktbeschreibungen, etwa von Reagenzien.

Warum es sich um drei Bücher handelt, ist nicht eindeutig geklärt. Vermutlich führte er zunächst parallel ein nach Stichworten gegliedertes Buch mit Literaturverweisen und eines für längere Protokolle und synoptische Darstellungen, bevor er ab Mai 1963, dem notierten Beginn des jüngsten Buches, ein weiteres hinzunahm. Obwohl die beiden älteren Bücher nicht eindeutig datierbar sind und die Einträge auf Literatur von der Vorkriegszeit bis ins Jahr 1965 verweisen, lässt sich aus dem Fabrikat abschätzen, dass sie in den frühen 1950er Jahren angeschafft und wohl auch begonnen wurden. ${ }^{6}$

Nachdem das kriegsbedingt in die Uckermark ausgelagerte Kaiser-Wilhelm-Institut für Zellphysiologie 1945 von der Roten Armee geplündert worden war und das Dahlemer Gebäude zunächst als Sitz des Oberkommandos der alliierten Streitkräfte diente, hatte Warburg seinen Institutsbetrieb um 1950 im Rahmen der Neugründung der MPG wieder in Dahlem eröffnen können und eine neue Generation Mitarbeiter eingestellt, $\mathrm{zu}$ der unter anderem die Labortechniker Hans-Siegfried Gewitz und Günter Krippahl gehörten. Krippahl, Jahrgang 1926, hatte nach dem Kriegseinsatz eine Ausbildung zum Fernmeldemonteur begonnen und arbeitete seit 1948 für Warburg. 1953, in der Zwischenzeit hatte er in der Abendschule einen Ingenieursabschluss erworben, stieg er zu Warburgs „persönlichem Assistenten“ auf und zeichnete seither als Mitautor auf diversen Veröffentlichungen zur Photosynthese. ${ }^{7}$ Warburg setzte also seine Arbeitsweise der Vorkriegszeit fort, mehrheitlich technische Assistenten als langfristige Mitarbeiter zu beschäftigen und nur mit wenigen Akademikern zusammenzuarbeiten. Viele dieser Techniker hatten zuvor im Bereich der Feinmechanik gearbeitet und wurden sowohl zur Geräte- und Methodenentwicklung als auch der Durchführung von Messungen eingesetzt (Abb. 3; Krebs 1979: 24 f.).

Die Leistungen Warburgs bis 1945 sind im Rahmen dieses Beitrags kaum darzustellen. Im Zusammenhang mit seinen grundlegenden Arbeiten zu Atmungsstoffwechsel, Photosynthese und Krebsforschung stand die Entwicklung manometrischer und photochemischer Techniken, und 1931 wurde ihm für die Arbeiten zu seinem „Atmungsferment“ der Nobelpreis für Physiologie oder Medizin verliehen (Henning 2004; Werner 1991). In den 1950er und 1960er Jahren veränderte sich Warburgs Rolle und Wahrnehmung allerdings, vor allem in Folge einer wissenschaftlichen Kontroverse zwischen ihm und verschiedenen Forschern in den USA (Nickelsen 2009). Gegenstand der Debatte waren Daten zur Quantenausbeute der Photosynthese, also dem Problem, wie viele Lichtquanten pro Molekül Chlorophyll benötigt werden, um eine photosynthetische Reaktion zu katalysieren. Bereits in den 1920er Jahren hatte Warburg durch den Einsatz manometrischer Techniken wie der Warburg-Apparatur und optischen Instrumenten wie Bolometer und Ulbrichtscher Kugel einen sehr niedrigen Wert für die Quantenausbeute 


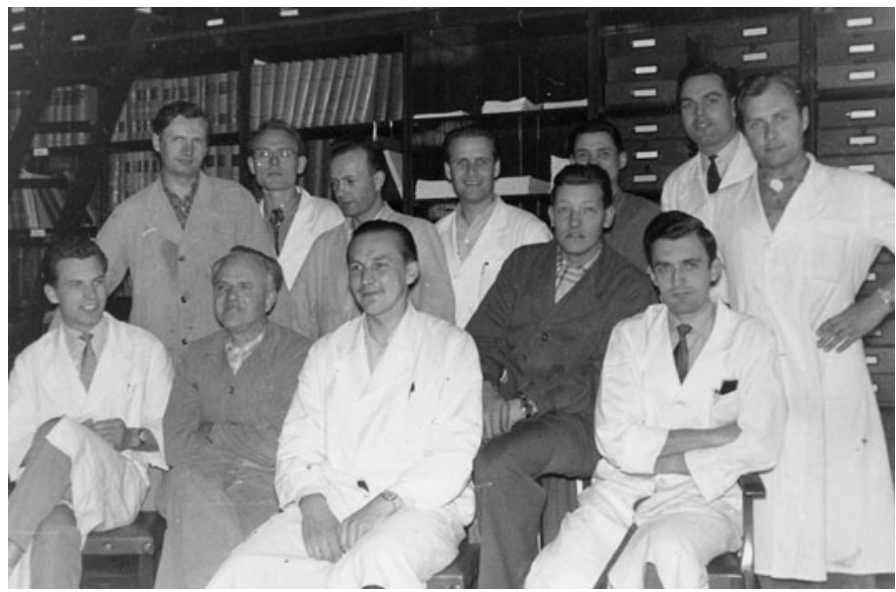

Abb. 3 Mitarbeiter des MPI für Zellphysiologie, 1961. Im dunklen Kittel in der rechten Bildmitte Günter Krippahl. ${ }^{8}$ Veröffentlicht mit freundlicher Genehmigung des Archivs der MPG, Berlin-Dahlem

ermittelt, der in den Folgejahren nicht bestätigt werden konnte. Da eine Ursache der Widersprüche in methodischen Details vermutet wurde, lud Warburgs ehemaliger Doktorand Robert Emerson ihn 1948 an die University of Illinois ein, um gemeinsam vor Ort Experimente durchzuführen. Entgegen der damit verbundenen Hoffnungen verschärfte sich aber der Konflikt in der Folgezeit und mündete in Polemiken und persönliche Angriffe. Warburg isolierte sich international zunehmend, und auch wenn das Institut für Zellphysiologie weiterhin aktiv publizierte, räumte sein Biograph Sir Hans Krebs ein, dass die meisten Arbeiten seit 1960 nicht mehr die „bahnbrechende Qualität" der vorangegangen Phase besessen hätten - sowohl die Photosyntheseforschung als auch Warburgs Theorie der Cancerogenese betreffend (Krebs 1979: 32-42, 69). Obgleich eine "Störung des Atmungsstoffwechsels“ als Krebsursache in der Fachwelt mehrheitlich auf Ablehnung traf, fanden Warburgs Thesen Resonanz in der deutschen Öffentlichkeit, wobei seine fortgesetzte Popularität auch darauf zurückzuführen sein mag, dass er einen Forschertypus verkörperte, der ansonsten weitgehend untergegangen zu sein schien. Abgesehen von einer kurzen Unterbrechung 1941 führte er das Dahlemer Institut seit 1932. ${ }^{9}$

Da Warburg mit der MPG eine Emeritierung nur auf eigenen Wunsch ausgehandelt hatte, blieb er nach seinem achtzigsten Geburtstag im Jahr 1963 Institutsdirektor, und erhielt sich als „Kaiser von Dahlem“ aus der Zeit gefallene Gewohnheiten wie einen Morgenritt im Innenhof seines Wohnhauses. ${ }^{10}$ Auch der Schriftverkehr zwischen dem Institut und der Generalverwaltung der MPG lässt einiges von der anachronistisch anmutenden Szenerie erkennen, welche diese Jahre geboten haben müssen: Warburg nutzte Treibhäuser und Gärten des Instituts wohl nicht nur, um Pflanzenmaterial für 
die Photosynthese-Forschung zu produzieren, sondern, in konsequenter Umsetzung seiner Hypothesen zur Krebsentstehung durch etwa Nahrungszusatzstoffe, ließ er dort auch Obst und Gemüse anbauen, und hielt Vieh - je nach Blickwinkel in verspäteter Gutsherrnmanier oder als urban gardener vor seiner Zeit (Henning 2004: 141; Werner 2000).

Während sich der ehemalige Dahlemer Campus der Kaiser-WilhelmGesellschaft mehr und mehr zum Sitz der Freien Universität wandelte, und John F. Kennedy 1963 den Henry-Ford-Bau vis-à-vis des Instituts für Zellphysiologie besuchte, wurde dort weiterhin die Grünalge Chlorella auf den Fenstersimsen kultiviert und führten Warburgs Techniker manometrische und optische Messreihen nach den etablierten Methoden durch. Günter Krippahls nun verfügbare Labortagebücher, ein geschlossener Bestand von 32 Bänden aus den Jahren 1952 bis 1970, führen diese Forschungsroutine eindrücklich vor Augen: Woche für Woche und Jahr um Jahr müssen Flüssigkulturen von Algen oder Präparationen aus etwa Kohlrabi und Spinat hergestellt worden sein, welche in die Glasgefäße der Warburg-Apparaturen gefüllt wurden, um dann über Stunden die Gasbilanzen zu protokollieren, und diese etwa mit der bolometrisch bestimmten Lichtabsorption der Zellen oder anderen physiologischen Parametern zu korrelieren. Noch im Monat vor Warburgs Tod am 1. August 1970, im Alter von 86 Jahren, finden sich Serien von Experimenten sowie handschriftliche Eintragungen Warburgs, und den Büchern zufolge muss die Arbeit auch wenige Wochen später wieder angelaufen sein. ${ }^{11}$

Bereits einige Jahre zuvor war mit der norwegischen Biochemikerin Birgit Vennesland eine von ihm und der MPG akzeptierte Nachfolgerin gefunden worden - doch im Mai 1970 kam es zum Zerwürfnis mit Warburg. Infolgedessen setzte die MPG nach seinem Tod den Direktor des Münchner Instituts für Zellchemie, Feodor Lynen, geschäftsführend ein, der Warburg kollegialfreundschaftlich verbunden gewesen war. ${ }^{12}$ In der Folgezeit traten jedoch die Konflikte zwischen Mitarbeitern, Jacob Heiß, dem Institutsverwalter und mutmaßlichen ehemaligen Lebensgefährten Warburgs und der MPG offen zu Tage. Zusätzlich zu der gescheiterten Nachfolgeregelung mit Birgit Vennesland, für die eine eigene Forschungsstelle eingerichtet wurde, war es vermutlich die anachronistische Position des Instituts in der Forschungslandschaft der 1970er Jahre, welche die MPG zu der Entscheidung veranlasste, dieses aufzulösen (Henning 2004: 141).

Der Schriftverkehr zwischen der MPG sowie Krippahl, Lynen und HeinzGünther Wittmann, Direktor am neu geschaffenen MPI für molekulare Genetik, informiert darüber, wie in dieser kritischen Phase über den Verbleib der Ressourcen verhandelt wurde. ${ }^{13}$ Und obwohl der Glasbläser aus Warburgs Werkstatt, Peter Ostendorf, sich Jahre später erinnern sollte, dass Institut sei „damals schon ein Museum" gewesen, schienen sowohl Instrumente wie Fertigkeiten durchaus auf Interesse zu stoßen. ${ }^{14}$ Während Ostendorf im nur 
einige hundert Meter entfernten MPI für molekulare Genetik angestellt wurde, wechselten andere wissenschaftliche Mitarbeiter an die „Forschungsstelle Vennesland“. ${ }^{15}$ Auch wenn eine vermutlich von Krippahl zusammengestellte Inventarliste zeigt, dass die Laborausstattung zum großen Teil aus den 1950er Jahren stammte, handschriftlich finden sich Vermerke wie „alt" oder „Schrott", waren die Interessenten keine Mittellosen. ${ }^{16}$ So informierte im Juli 1972 die Generalverwaltung der MPG Wittmann, dass Lynen bereits einige Wünsche geäußert und Birgit Vennesland sowie Benno Hess einiges erhalten hätten - das Inventar werde nun ihm und dem ebenfalls benachbarten Fritz-Haber-Institut zugehen. Hess, Direktor am Dortmunder MPI für Ernährungsphysiologie, übernahm ein Bolometer und eine Ulbricht'sche Kugel, und Lynen ließ die nun aufgefundenen Instrumente nach Martinsried überführen, wohin seine Abteilung umgezogen war. Dieter Oesterhelt erinnerte sich, zu diesem Zweck als Teil einer Münchner Delegation nach Berlin gereist zu sein. ${ }^{17}$ Wichtiger als der Transfer von Instrumenten war sicherlich, dass Lynen auch Günter Krippahl in seine Abteilung übernahm, den Ostendorf als „rechte Hand“ Warburgs bezeichnete. ${ }^{18}$ Hans-Siegfried Gewitz, in einer Personalübersicht vom März 1972 ebenfalls als „wissenschaftlicher Assistent “ aufgeführt - so die offizielle Bezeichnung für Warburgs wissenschaftlich arbeitende Techniker - wechselte zunächst an die Forschungsstelle Vennesland und später zur Abteilung Wittmann am MPI für molekulare Genetik, wo er gemeinsam mit einem Doktoranden namens HansJörg Rheinberger pipettierte. ${ }^{19}$

\section{Günter Krippahl am MPI für Biochemie oder das Alte im Neuen, 1972-1994}

Dieter Oesterhelt war im Herbst 1970 nach einem Sabbatical in San Francisco an das Biochemische Institut der Ludwig-Maximilians-Universität zurückgekehrt, welches in der Karlstraße nahe dem Münchner Hauptbahnhof angesiedelt war. Die Position eines akademischen Rates sicherte ihm zwar eine gewisse Autonomie, aber nicht die instrumentellen Ressourcen, über die er zuvor als Mitarbeiter in Lynens MPI für Zellchemie verfügt hatte. ${ }^{20}$ Lynen, der auch einen Lehrstuhl an der Universität bekleidete, beklagte selbst, dass der Universität, die bis dahin unter einem Dach mit dem MPI untergebracht gewesen war, nach dessen Auszug 1972 nur wenige Gerätschaften geblieben seien (Will 2011). So ist es nicht verwunderlich, dass Oesterhelt den Kontakt zu Lynen auch nutzte, um in dessen neuen Labors Experimente durchzuführen. Und obwohl Lynen nach Oesterhelts Erinnerung anfangs nicht viel von dessen neuartigen Untersuchungen an einem dem Sehpigment Rhodopsin ähnlichen Protein aus halophilen - salzliebenden - Mikroben hielt, 
und das Thema in der Tat einen gewissen Bruch mit einer an der organischen Chemie und Enzymologie orientierten Forschung mit sich bringen sollte, willigte er offensichtlich ein, Krippahl in Martinsried für Arbeiten an Halobakterien einzusetzen.

In Oesterhelts Arbeit standen sich nicht nur etablierte und neuartige Themen gegenüber, sondern auch traditionelle und technologisch avancierte Methoden: Neben automatisierten Aminosäurebestimmungen von Proteinen führte er klassische Nachweisreaktionen zur Charakterisierung chemischer Bindungen durch, und neben spektroskopischen Messungen mit Benno Hess in Dortmund standen die Arbeiten an der Warburg-Apparatur, für die regelmäßig Zellsuspensionen nach Martinsried geschafft wurden. Die Originaldaten dieser Studien stellen von Krippahl mit Zahlenkolonnen zu Gasverbrauch und Lichtabsorption der Zellen eng beschriebene Blätter dar. In der 1973 daraus hervorgegangenen Veröffentlichung wirkt die Arbeitsweise von Warburgs Institut nicht nur in den verwendeten Instrumenten und der Herangehensweise fort, sondern auch in der Tatsache, dass ein technischer Assistent als Mitautor zeichnete. ${ }^{21}$

Ziel des Vorhabens von Oesterhelt und Krippahl war es, Daten zur physiologischen Funktion der das Bakteriorhodopsin enthaltenden „Purpurmembran“ von Halobakterien zu gewinnen. Interessanterweise wurden in San Francisco zunächst molekulare Struktur und chemische Zusammensetzung dieses leuchtend gefärbten Bestandteiles der Zellmembran charakterisiert, die biologisch zentrale Frage aber, wozu ein dem Sehrezeptor ähnliches Protein dem Mikroorganismus diene, blieb offen (Grote 2012).

Es scheint, als seien in San Francisco wie auch in München erst ab 1972 systematisch funktionelle, physiologische Experimente begonnen worden: Während vorher das biochemisch präparierte Membranmaterial als Substrat von Experimenten in vitro diente, wurde nun die Rolle der Purpurmembran im Stoffwechsel lebender Zellen untersucht. Auch wenn Krippahls Ankunft in Martinsried nicht Ursache dieser veränderten Experimentalstrategie gewesen ist, da andere Arbeiten auf der Zellebene vermutlich etwas früher begonnen wurden, veränderte die Inkorporation von Warburgs physiologischem Instrumentarium das Projekt um die Purpurmembran doch signifikant.

Die Grundaussage der Studie von Oesterhelt und Krippahl lässt sich dergestalt zusammenfassen, dass eine Belichtung von die Purpurmembran enthaltenden Halobakterien deren Sauerstoffverbrauch und damit Atmungsaktivität senkt, was als Hinweis darauf verstanden wurde, dass die Purpurmembran im Stoffwechsel der Zelle eine energieliefernde, dem Chlorophyll ähnliche Funktion besitzt. Während der Gasverbrauch von Zellsuspensionen mit einer Warburg-Apparatur gemessen wurde, setzten die Autoren Bolometer und Ulbricht'sche Kugel ein, um die Quantenausbeute der durch die Purpurmembran getriebenen, neuartigen photosynthetischen Reaktion zu bestimmen (vgl. Abb. 2, zu diesen Instrumenten siehe auch Anmerkung 4). 
Trotz der Kontroverse um die Quantenausbeute der Photosynthese scheint es, als habe der erneute Einsatz der alten Warburg'schen Methoden relevante und anerkannte Daten geliefert. So wurden die Messungen Krippahls zur „Atmungshemmung" durch die Purpurmembran auch in einer gemeinsamen Publikation in den renommierten Proceedings of the National Academy of Sciences of the USA untergebracht, welche die bioenergetische Funktion der Purpurmembran als photocoupler neben die Thylakoidmembran der Chloroplasten stellte und die zu den wichtigsten des neu entstehenden Feldes zählt. Messungen zur Quantenausbeute der Purpurmembran wurden gemeinsam mit Benno Hess publiziert. ${ }^{22}$

Bereits kurz bevor Feodor Lynen 1979 überraschend verstarb, war Oesterhelt zum Leiter der neu geschaffenen Abteilung „Membranbiochemie“ am Martinsrieder Institut ernannt worden. Nun übernahm er nicht nur Krippahl, sondern auch die Dahlemer Instrumente. Obgleich seit den 1970er Jahren Elektroden zur Messung von Gaskonzentrationen die WarburgApparatur zunehmend ablösten, wurde diese und die optischen Instrumente weiter genutzt - vielleicht, weil sie verlässlich unter verschiedenen Bedingungen arbeiteten, weil die Ergebnisse besser mit bekannten Werten vergleichbar waren, oder schlicht weil sie existierten und jemand sie $\mathrm{zu}$ bedienen wusste. Krippahl zeichnet als Mitautor von mindestens drei weiteren Publikationen aus Oesterhelts Abteilung, deren letzte auf 1992 datiert, und zu denen er jeweils Zellwachstumsversuche oder optische Experimente beitrug. Als sich 1986 zwei Forscher bei ihm für Messungen mit der WarburgApparatur bedankten, hatte das Londoner Science Museum bereits Teile der Ausrüstung aus Martinsried übernommen, die dort als „German, midtwentieth century“ klassifiziert werden. ${ }^{23}$

Darüber hinaus bekleidete Krippahl offenbar eine wichtige Rolle bei Organisation und Unterhalt der Abteilung, die bis zu dreißig aktive Forscher umfasste. Als er nach 43 Jahren Anstellung innerhalb der MPG im August 1991 in den Ruhestand trat, bat Oesterhelt die Verwaltung des Instituts, ihm seine Dienstwohnung in Martinsried weiterhin zu gewähren, damit er seine Aufgaben in Teilzeitbeschäftigung fortsetzen könne. Und als Günter Krippahl im November 1994 verstarb, schrieb Oesterhelt in einer Traueranzeige, dieser habe „Apparate und Menschen in seiner beharrlichen, gerechten, immer kurzen Art [betreut]. “24

\section{Alte Instrumente, bewährte Techniker - Forschung als Dienst und Beständigkeit?}

Ob Krippahls und Warburgs Bücher es ermöglichen könnten, die Kontroverse um den Quantenbedarf der Photosynthese historisch weiter aufzuarbeiten, 
muss eine detailliertere Untersuchung zeigen. Immerhin war es zu keinem geringen Teil Krippahl, der über zwei Dekaden jene Messungen durchführte, die Gegenstand des Streits waren, und die erhaltenen Bände scheinen geradezu pedantisch geführt. Darüber hinaus könnten die „Kochbücher“ Warburgs weiteren Einblick in dessen Arbeiten der Nachkriegszeit geben. Gegenüber chronologischen Experimentalaufzeichnungen oder Notizen stellen derartige Protokoll- oder Referenzbücher eine oft kollektive Verschriftlichung der Routinen und Kniffe eines Labors dar und dürften in chemischen wie lebenswissenschaftlichen Labors eine wichtige Funktion zur Ausprägung einer Laboridentität besessen haben (Rheinberger 2005: 353 f.).

Im Kontext der Geschichte der molekularen Lebenswissenschaften illustriert der Fund das Fortwirken einer antiquiert erscheinenden wissenschaftlichen Methode in der jüngsten Vergangenheit. Auch wenn es sich hier um einen Einzelfall handelt, wäre zu prüfen, ob Warburg-Apparaturen nicht doch länger eingesetzt wurden als bisher angenommen. Immerhin produzierte der Laborgerätehersteller B. Braun Melsungen bis Mitte der 1980er Jahre solche Geräte. Selbst als diese zu einem Auslaufmodell der Forschung geworden waren, könnten sie aufgrund ihrer Robustheit und Anschaulichkeit in der akademischen Lehre durchaus noch eine Rolle gespielt haben. $^{25}$

Ohne die Dimensionen dieses oder anderer Beispiele hier abschließend einschätzen zu können, erschiene es doch einen Versuch wert, die erfolgreiche Weiternutzung einer einfachen Technologie im Lichte dessen zu begreifen, was David Edgerton programmatisch als Shock of the Old bezeichnet hat (Edgerton 2008). Es wäre also auch mit Blick auf die Laborforschung eine weniger innovations- als nutzungszentrierte Perspektive einzunehmen, und etwa die Verteilung von einfachen oder etablierten Instrumenten sowie deren tatsächlicher Einfluss zu untersuchen. Als Ursachen von instrumentellen Kontinuitäten, die parallele Neuentwicklungen und Modernisierungen nicht ausschließen, könnten Kosten, die Lebensdauer von Gerätschaften, Wissen um deren Bedienung, Spezialanfertigungen, eine Vergleichbarkeit von Ergebnissen oder das Vertrauen in das Bewährte in Betracht gezogen werden never change a running system.

Neben der Fortdauer einer alten Technologie heben Krippahls und Warburgs Bücher einen weiteren eher unspektakulären Aspekt der wissenschaftlichen Praxis hervor - denken wir noch einmal an das Protokoll zur Herstellung des Coenzyms DPN durch wiederholtes Mischen, Trennen und Extrahieren von Substanzen. Ebenso wie derartige präparative Arbeiten scheint auch die in Krippahls Labortagebüchern dokumentierte Forschung selbst durch wiederholtes Ausführen und Variieren einfacher Schemata gekennzeichnet, das heißt, eher durch Handarbeit, Ablesen, Notieren von Messwerten und Berechnungen als durch Innovationen im Sinne neuer Technologien oder Darstellungsformen. Damit, und dies bringt uns noch 
einmal zur Figur des Labortechnikers, ist ein weiterer Aspekt angedeutet, unter dem das Verhältnis von Kontinuität und Wandel in den Lebenswissenschaften des 20. Jahrhunderts erneut betrachtet werden könnte.

Günter Krippahl verbrachte mehr als vierzig Jahre in Dahlem und Martinsried zwischen Schüttelkulturen diverser Zellen, Chemikalien, Zentrifugen, Abwaschbecken, Thermostaten und Kinolampen. Auch wenn man diese Kontinuität mit der Außergewöhnlichkeit Warburgs erklären könnte, zeigt sich hier doch ein allgemeines Phänomen: Während Promovierende und Wissenschaftler, jene, die sichtbar neue Verfahren entwickeln und Ergebnisse produzieren, während also das Personal der Innovation schnell wechselt, erfordert ein funktionierendes Labor auch ein großes Maß an Erhaltungs- und Wartungsarbeit, oder maintenance, um eines von Edgertons Schlagworten aufzugreifen. Insofern besitzen Laborassistenten oder Techniker nicht nur eine wichtige, unter Umständen entscheidende Funktion in der Experimentalpraxis, sondern auch eine besondere soziale Rolle. ${ }^{26}$ Im Unterschied zu dem von Steven Shapin (2008) beschriebenen, vorherrschenden Typus des „unsichtbaren Labortechnikers" waren Warburgs Assistenten aber durchaus sichtbar - er führte diese stets als Mitautoren, beteiligte sie an Patenten, und förderte ihre Weiterbildung. Erwin Negelein beispielsweise begann nach einer Feinmechanikerausbildung bei Siemens \& Halske 1919 in Warburgs Abteilung, schloss berufsbegleitend Studium und Promotion der Chemie ab und wurde in der Nachkriegszeit Professor an der Humboldt-Universität und Institutsdirektor in Berlin-Buch (Eichhorst 2000). Es wäre zweifellos interessant, diese besondere Konturierung des Labortechnikers im Institut für Zellphysiologie mit Blick auf Warburgs Charakter und Verständnis von Wissenschaft zu untersuchen.

Im Lichte eines vielfach auf Kreativität, Neuerung und Wandel zentrierten Verständnisses der rezenten Lebenswissenschaften erinnern die Langlebigkeit von Techniker und Instrument, wie auch die in diesem Fund sichtbaren profanen Aspekte der Forschungspraxis daran, wie sehr auch die molekularen Lebenswissenschaften von Handarbeit und strenger Organisation, von Routine und Kontinuität bestimmt wurden und vermutlich noch werden.

\section{Danksagung}

Ich danke Eleonore Haack und Dieter Oesterhelt für den freundlichen Empfang und die vielfachen Hilfestellungen während meiner Aufenthalte in Martinried, wie auch für die Einsicht in unveröffentlichte Dokumente. Robert Meunier, Kärin Nickelsen, Hans-Jörg Rheinberger, Max Stadler und Elmar Zeitler, Mitarbeitern des Archivs der MPG und der Berlin-Brandenburgischen Akademie der Wissenschaften, des Science Museum London und der Firma B. Braun Melsungen sowie Kolloquiumsteilnehmern an der HU und TU Berlin 
danke ich für Gespräche, Hinweise und Diskussionen. Die Arbeit an diesem Projekt wurde durch die Deutsche Forschungsgemeinschaft gefördert (DFGKennzeichen GR 3835/1-1).

\section{Anmerkungen}

1 So der ehemalige Präsident der MPG Reimar Lüst, zitiert nach Henning 2004: 141.

2 Das ehemalige Kaiser-Wilhelm-Institut für Zellphysiologie beherbergte seit 1932 Warburg und seine Mitarbeiter. Im Vordergrund der Institutsgarten, welcher seit den Nachkriegsjahren auch zum Anbau von Nutzpflanzen genutzt wurde. Das MPI für Biochemie zentralisierte ab 1972 die früheren Einzelinstitute für Lederforschung, Zellchemie und Biochemie auf damals unbebautem Gelände im Süden Münchens. In den frei um ein Zentrum angeordneten Gebäudeflügel forschten Arbeitsgruppen zu biochemischen, biophysikalischen und medizinischen Themen, vgl. Heßler 2007.

3 Persönliche Mitteilung Dieter Oesterhelts bei einem Besuch des Autors im Mai 2012. Die Bücher wurden mittlerweile an das Archiv der MPG in Berlin-Dahlem und die Instrumente an das Deutsche Museum in München übergeben.

4 Die Innenseite der Ulbrichtschen Kugel ist mit einer totalreflektierenden Farbe bestrichen, so dass durch eine Öffnung eingestrahlte und nicht von der dort eingebrachten Probe (etwa eine Flüssigkultur von der Grünalge Chlorella) absorbierte Lichtstrahlen von dem an einer zweiten Öffnung angebrachten Kohlefaden des Bolometers (kleiner schwarzer Kasten mit Aufsatz in der Bildmitte) absorbiert werden. Die so verursachte Leitfähigkeitsänderung des Kohlefadens ermöglicht die Quantifizierung von Strahlungsintensitäten - ein Verfahren, das von Emil Warburg an der Physikalisch-Technischen Reichanstalt entwickelt und unter anderem von Max Planck eingesetzt wurde. Einen derartigen Aufbau verwandten Benno Hess, Günter Krippahl und Dieter Oesterhelt 1972-1973 zur Untersuchung der das Bakteriorhodopsin enthaltenden Purpurmembran von Halobakterien.

5 Archiv der MPG, Berlin, II. Abt. Rep. 16 Nachlass MPI für Zellphysiologie Nr. 30 (Buch 1).

6 Die Bücher tragen einen Aufkleber der Berliner Papierwarenhandlung „Reuter \& Siecke“, der sich auch in den Bänden 5-16 der chronologisch geführten Labortagebücher Warburgs findet. Diese decken den Zeitraum von März 1950 bis Januar 1954 ab. Siehe Archiv der Berlin-Brandenburgischen Akademie der Wissenschaften (BBAW), Nachlass Otto Heinrich Warburg, Lfd. Nr. 5-16.

7 Lebenslauf Günter Krippahl, Personalmappe G. K., MPI für Biochemie, Martinsried.

8 Von den zwölf abgebildeten Personen stellen acht wissenschaftlich arbeitende Labortechniker dar, die Warburg als Mitautoren führte.

9 Nickelsen/Govindjee 2011: 123 f. Zu Warburgs ungewöhnlicher Stellung im Nationalsozialismus siehe Henning 2004: 133-136, sowie Nickelsen 2008.

10 Henning 2004. Persönliche Mitteilung Wolfgang Lefèvre, Berlin.

11 Labortagebücher Krippahl, Archiv der MPG, II. Abt. Rep. 16, MPI für Zellphysiologie, Nr. 16-18; Labortagebuch Warburg, Archiv BBAW, NL O. H. Warburg, Lfd. Nr. 62.

$12 \mathrm{Zu}$ Feodor Lynen siehe Will 2011. Siehe auch die Korrespondenz zwischen Lynen und Warburg, Archiv BBAW, NL O. H. Warburg, Lfd. Nr. 611 und 1117.

13 Vermerk „Betr.: Auflösung des Instituts für Zellphysiologie“, 5. Juli 1972; Brief Roeske an Krippahl, 5. Juli 1972, beides Archiv der MPG, II. Abt., Rep. I A Institutsbetreuerakten MPI für Zellphysiologie, II. 03-03 Zellphys.

14 Siehe das Interview mit Peter Ostendorf, „Das war damals schon ein Museum“, in: Gruss/ Rürup, Hg., 2010: 252-254.

15 Anlage zum Sozialplan für die Mitarbeiter des MPI für Zellphysiologie, Archiv der MPG, II. Abt. Rep. 16 MPI für Zellphysiologie, Nr. 39.

16 Inventarliste (undatiert), Brief der Generalverwaltung der MPG an Wittmann, 5. Juli 1972, Archiv der MPG, II. Abt., Rep. 16, MPI für Zellphysiologie, Nr. 37, 38. 
17 Persönliche Mitteilung, Dieter Oesterhelt, 17. Februar 2012.

18 Interview mit mit Peter Ostendorf, „Das war damals schon ein Museum“, in: Gruss/ Rürup, Hg., 2010: 254.

19 Anlage zum Sozialplan für die Mitarbeiter des MPI für Zellphysiologie, Archiv der MPG, II. Abt. Rep. 16 MPI für Zellphysiologie, Nr. 39. Persönliche Mitteilung Hans-Jörg Rheinberger, 1. August 2012. Auch Gewitz wurde weiterhin als Mitautor auf Publikationen genannt, siehe etwa Schnier u. a. 1990.

20 Lebenslauf Dieter Oesterhelt, persönliche Sammlung.

21 Labornotizen Dieter Oesterhelt „Krippahl“, Abteilung MPI für Biochemie, Martinsried. Diese Dokumente wurden vor Ort analysiert und werden ins Archiv der MPG überstellt; Oesterhelt/Krippahl 1973.

22 Oesterhelt/Hess 1973, Oesterhelt/Stoeckenius 1973, siehe auch Grote 2012. Eine Vitrine mit Warburg-Memorabilien im Archiv der MPG stellt unter anderem das von Benno Hess übernommene Bolometer aus.

23 Z. B. Lanyi/Tittor/Váró/Krippahl/Oesterhelt 1992, Wagner/Oesterhelt/Krippahl/Lanyi 1981. Duschl/Wagner 1986 bedankten sich bei Krippahl; persönliche Mitteilung von John Underwood (Science Museum London), 28. Juni 2012.

24 Oesterhelt an Krell, 2. September 1991; Traueranzeige Günter Krippahl 4. November 1994 (vermutlich zum Aushang im Institut), beides Personalmappe Günter Krippahl, MPI für Biochemie, Martinsried.

25 E-Mail Wolfgang Koller (Unternehmensarchiv B. Braun, Melsungen), 12. Juni 2012. Im mikrobiologischen Praktikum der Universität Göttingen etwa fand die Apparatur bis Ende der 1990er Jahre Verwendung (persönliche Mitteilung Kärin Nickelsen, 4. Mai 2012). Als Graßhoff/Casties/Nickelsen 2000 eine historische Rekonstruktion der Krebs-HenseleitExperimente zum Harnstoffzyklus planten, erfuhren sie, dass die ETH Zürich das letzte Exemplar 1999 verschrottet hatte.

26 Zu Laborassistenten, Mechanikern und Zeichnern in den physikalischen Wissenschaften, vgl. Hentschel, Hg., 2008.

\section{Literatur}

Duschl, Albert/Wagner, Gottfried, 1986. Primary and Secondary Chloride Transport in Halobacterium halobium. Journal of Bacteriology, 168, 548-552.

Edgerton, David H. E., 2008. The Shock of the Old. Technology and Global History since 1900. London: Profile.

Eichhorst, Thomas, 2000. Vom Mechaniker zum Professor. Zum Wirken des Biochemikers Erwin Negelein (1897-1979), Dissertation, Medizinische Fakultät Charité der HumboldtUniversität zu Berlin.

Graßhoff, Gerd/Casties, Robert/Nickelsen, Kärin, 2000. Zur Theorie des Experiments. Untersuchungen am Beispiel der Entdeckung des Harnstoffzyklus. Bern: Bern Studies in the History and Philosophy of Science.

Grote, Mathias, 2012. Purple Matter, Membranes and ,Molecular Pumps' in Rhodopsin Research, Journal for the History of Biology, published ahead of print as DOI 10.1007/s10739012-9333-9.

Gruss, Peter/Rürup, Reinhard, Hg., 2010. Denkorte. Max-Planck-Gesellschaft und KaiserWilhelm-Gesellschaft. Brüche, Kontinuitäten, Erinnerungen 1911-2011. Dresden: Sandstein Verlag.

Henning, Eckart, 2004. Otto Heinrich Warburg - der ,Kaiser von Dahlem‘. Veröffentlichungen aus dem Archiv der Max-Planck-Gesellschaft. Beiträge zur Wissenschaftsgeschichte Dahlems, 13, 125-146.

Hentschel, Klaus, Hg., 2008. Unsichtbare Hände. Zur Rolle von Laborassistenten, Mechanikern, Zeichnern u.a. Amanuenses in der physikalischen Forschungs- und Entwicklungsarbeit. Diepholz u. a.: Verlag für Geschichte der Naturwissenschaft und Technik.

Heßler, Martina, 2007. Die kreative Stadt. Zur Neuerfindung eines Topos. Bielefeld: Transcript. 
Krebs, Hans, 1979. Otto Warburg. Zellphysiologe, Biochemiker, Mediziner. Darmstadt: Wissenschaftliche Verlags-Gesellschaft.

Lanyi, Janos K./Tittor, Jörg/Váró, György/Krippahl, Günter/Oesterhelt, Dieter, 1992. Influence of the Size and Protonation State of Acidic Residue 85 on the Absorption Spectrum and Photoreaction of the Bacteriorhodopsin Chromophore. Biochimica et Biophysica Acta (BBA) - Bioenergetics 1099, 102-110.

Morange, Michel, 1998. A History of Molecular Biology. Cambridge (Mass.) u. a.: Harvard University Press.

Nickelsen, Kärin, 2008. Ein bisher unbekanntes Zeitzeugnis. Otto Warburgs Tagebuchnotizen von Februar-April 1945. NTM Zeitschrift für Geschichte der Wissenschaften, Technik und Medizin 16, 103-115.

Nickelsen, Kärin, 2009. Of Light and Darkness. Modelling Photosynthesis 1840-1960. Habilitationsschrift, Universität Bern.

Nickelsen, Kärin/Govindjee, 2011. The Maximum Quantum Yield Controversy. Otto Warburg and the „Midwest-Gang“. Bern: Bern Studies in the History and Philosophy of Science.

Oesterhelt, Dieter/Hess, Benno, 1973. Reversible Photolysis of the Purple Complex in the Purple Membrane of Halobacterium halobium. European Journal of Biochemistry 37, 316-326.

Oesterhelt, Dieter/Krippahl, Günter, 1973. Light Inhibition of Respiration in Halobacterium halobium. FEBS Letters 36, 72-76.

Oesterhelt, Dieter/Stoeckenius, Walther, 1973. Functions of a New Photoreceptor Membrane. Proceedings of the National Academy of Sciences of the United States of America 70, 28532857.

Olby, Robert, 1990. The Molecular Revolution in Biology. In: Robert C. Olby/Geoffrey N. Cantor/ John R.R. Christie/M. Jonathan S. Hodge, Hg., Companion to the History of Modern Science. London u. a.: Routledge, 503-520.

Rheinberger, Hans-Jörg, 2005. Kritzel und Schnipsel. In: Dotzler, Bernhard J./Weigel, Sigrid, Hg., "fülle der combination". Literaturforschung und Wissenschaftsgeschichte, München: Wilhelm Fink Verlag, 343-356.

Schnier, Joachim u. a., 1990. Isolation and Characterization of Bacillus stearothermophilus $30 \mathrm{~S}$ and 50S Ribosomal Protein Mutations. Journal of Bacteriology, 172, 7306-7309.

Shapin, Steven, 2008. Unsichtbare Labortechniker. In: Hentschel, Klaus, Hg., Unsichtbare Hände. Zur Rolle von Laborassistenten, Mechanikern, Zeichnern u. a. Amanuenses in der physikalischen Forschungs- und Entwicklungsarbeit. Diepholz u. a.: Verlag für Geschichte der Naturwissenschaft und Technik, 26-44.

Wagner, Gottfried/Oesterhelt, Dieter/Krippahl, Günter/Lanyi, Janos K., 1981. Bioenergetic Role of Halorhodopsin in Halobacterium halobium Cells. FEBS Letters 131, 341-345.

Werner, Petra, Hg., 1991. Ein Genie irrt seltener - Otto Heinrich Warburg: ein Lebensbild in Dokumenten. Berlin: Akademie Verlag.

Werner, Petra, 2000. „Das Brot dem Zugriff des Chemikers entziehen“ - Otto Warburgs Korrespondenz zu Umweltfragen. In: Wessel, Karl-Friedrich/Schulz, Jörg /Hackethal, Sabine, Hg., Ein Leben für die Biologie(geschichte), Festschrift zum 75. Geburtstag von Ilse Jahn, Bielefeld: Kleine, 190-204.

Will, Heike, 2011. Sei naiv und mach' ein Experiment: Feodor Lynen: Biographie des Münchner Biochemikers und Nobelpreisträgers. Weinheim: VCH.

\section{Mathias Grote}

Institut für Philosophie, Literatur-

Wissenschafts- und Technikgeschichte

Technische Universität Berlin

Straße des 17. Juni 135

10623 Berlin

Deutschland

E-Mail: mathias.grote@tu-berlin.de 